OLIVEIRA, A.P.; ANDRADE, A.C.; TAVARES SOBRINHO, J.; PEIXOTO, N. Avaliação de linhagens e cultivares de feijão-vagem, de crescimento indeterminado, no município de Areia-PB. Horticultura Brasileira, Brasília, v. 19, n. 2, p. 159-162, julho 2.001.

\title{
Avaliação de linhagens e cultivares de feijão-vagem de crescimento indeterminado, no município de Areia-PB.
}

\author{
Ademar P. Oliveira ${ }^{1}$; Adriano C. Andrade'; José Tavares Sobrinho'; Nei Peixoto \\ ${ }^{1}$ UFPB, C. Postal 02, 58.397-000 Areia - PB.; ${ }^{2}$ Agência Goiana de Desenvolvimento Rural e Fundiário, C. Postal 608, 75.001-970 \\ Anápolis-GO. e-mail: ademar@cca.ufpb.br
}

\section{RESUMO}

Avaliaram-se quinze linhagens (Hav 13, Hav 14, Hav 21, Hav 22, Hav 25, Hav 38, Hav 40, Hav 41, Hav 49, Hav 53, Hav 56, Hav 60, Hav 65, Hav 67 e Hav 68) e oito cultivares comerciais (Macarrão Favorito Ag 480, Macarrão Preferido Ag 482, Manteiga Maravilha Ag 481, Teresópolis Ag 484, Macarrão Bragança, Macarrão Trepador Topseed, Macarrão Trepador Hortivale e Macarrão Trepador ISLA) de feijão-vagem de crescimento indeterminado. $\mathrm{O}$ ensaio foi conduzido na Universidade Federal da Paraíba, em Areia, no período de março a agosto de 1999 em Latossolo VermelhoAmarelo. As linhagens Hav 22 (31,6 t/ha), Hav 38 (35,00t/ha), Hav 41 (31,5 t/ha), Hav 68 (30,5 t/ha), e as cultivares Macarrão Favorito $\operatorname{Ag} 480$ (30,3 t/ha), Macarrão Preferido Ag 482 (39,1 t/ha) e Manteiga Maravilha Ag 481 (36,2 t/ha) destacaram-se por apresentarem alta produtividade, peso médio de vagens de acordo com a preferência do mercado local, e teor de fibra dentro dos padrões comerciais. A cultivar Teresópolis Ag 484 (58,5 t/ha), embora tenha apresentado alta produtividade, necessita de estudos de mercado, para que possa ser indicada para os produtores, uma vez que apresenta vagens grandes e achatadas.

\begin{abstract}
Evaluation of breeding lines and cultivars of climbing snap beans in Paraiba, Brazil.

Fifteen breeding lines (Hav 13; Hav 14; Hav 21; Hav 22; Hav 25; Hav 38; Hav 40; Hav 41; Hav 49; Hav 53; Hav 56; Hav 60; Hav 65; Hav 67 and Hav 68) and eight cultivars (Macarrão Favorito Ag 480; Macarrão Preferido Ag 482; Manteiga Maravilha Ag 481; Teresópolis Ag 484; Macarrão Bragança; Macarrão Trepador Topseed; Macarrão Trepador Hortivale and Macarrão Trepador ISLA) of climbing snap beans were evaluated under field conditions in Areia, State of Paraiba, Brazil. The trial was carried out from March to August, 1999. The best results (high yield, good average weight and low fiber content of pods) were obtained with the breeding lines Hav 22 (31.6 t/ha); Hav 38 (35.0 t/ha); Hav 41 (31.5 t/ha) and Hav 68 (30.5 t/ha), and with the cultivars Macarrão Favorito Ag 480 (30.3 t/ha); Macarrão Preferido $\operatorname{Ag} 482$ (39.1 t/ha) and Manteiga Maravilha Ag 481 (36.2 t/ha). Cultivar Teresópolis Ag 484 (58.5 t/ ha) presented the highest yield. However, the pods were flattened and long; so additional studies are necessary to evaluate the local market preference.
\end{abstract}

Palavras-chave: Phaseolus vulgaris L, produtividade.

Keywords: Phaseolus vulgaris L, yield.

(Aceito para publicação em 03 de abril de 2.001)

A produção de feijão-vagem na Paraíba, como em todo o Brasil, é conduzida por pequenos produtores, utilizando principalmente cultivares de crescimento indeterminado; A produção destina-se ao consumo fresco e em pequenas quantidades à industrialização (Viggiano, 1990; Peixoto et al., 1997; Hamasaki et al., 1998).

É crescente o cultivo do feijão-vagem na região de Areia, convertendo-se em uma das principais hortaliças cultivadas na região, sendo as cultivares Macarrão Trepador Topseed e Macarrão Trepador Hortivale as mais utilizadas pelos produtores. Nesta região o período chuvoso abrange os meses de março a agosto, seguido do período de estiagem que vai de setembro a fevereiro, quando ocorrem elevadas temperaturas, com média anual de $23^{\circ} \mathrm{C}$, e baixa umidade relativa do ar. $\mathrm{O}$ desenvolvimento do feijão-vagem é favorecido quando a temperatura ambiente varia entre 18 e $30^{\circ} \mathrm{C}$. (Blanco et al., 1997), podendo adaptar-se bem a climas frescos ou quentes com temperaturas variando entre 18 e $50^{\circ} \mathrm{C}$ (Nadal et al., 1986). Deste modo, as características climáticas de Areia são satisfatórias para sua expansão.

Existem no mercado brasileiro cultivares de boa aceitação comercial. Entretanto, não há um programa nacional de avaliação e recomendação de cultivares que poderia resultar na utilização das mais adaptadas a cada ambiente específico. Estudos sobre novas opções são necessários pois o produtor normalmente tem utilizado qualquer semente disponível no mercado. A indicação de cultivares apropriadas proporciona maior segurança aos produtores, inclusive facilitando a obtenção de crédito e aceitação do produto no mercado (Hamasaki et al., 1998).
Têm sido escassos os trabalhos de melhoramento de feijão-vagem no Brasil e as cultivares disponíveis são utilizadas nas diversas regiões, sem levar em consideração as possíveis diferenças de comportamento em ambientes diversos. Assim o estudo da interação genótipo $\mathrm{x}$ ambiente possibilita a recomendação de cultivares, considerando-se a adaptabilidade e estabilidade em relação às diferentes condições de cultivo. Esta avaliação constitui-se numa importante ferramenta na recomendação de cultivares. Para a empresa produtora de sementes interessam cultivares estáveis que possam ser cultivadas em diferentes ambientes, enquanto que para o produtor seria desejável a utilização de cultivares adaptadas às suas condições edafoclimáticas e a tecnologia específica de produção (Peixoto et al., 1993).

Para a escolha de um novo genótipo a ser plantado em determinado local, é 
sempre desejável que existam ensaios visando a seleção dos mais adaptados. Recomendam-se inicialmente, plantios em escala experimental e, somente após obtidos resultados animadores, deverão ser feitos plantios em maior escala, com o novo genótipo. Este método é um requisito importante, para a indicação de novas cultivares de qualquer hortaliça, pois o comportamento de cada genótipo depende do ambiente como um todo principalmente, do clima e do solo (Filgueira, 1982).

Este trabalho teve como objetivo avaliar o comportamento de linhagens de feijão-vagem, obtidas em programas nacionais de melhoramento genético, e cultivares existentes no mercado, nas condições do município de Areia.

\section{MATERIAL E MÉTODOS}

O trabalho foi conduzido em campo experimental da Universidade Federal da Paraíba, em Areia, em solo Latossolo Vermelho-Amarelo, no período de março a agosto de 1999.

$\mathrm{A}$ análise química da área experimental resultou: $\mathrm{pH}_{2} \mathrm{O}=6,90$; $\mathrm{P}$ disponível = 156,00 mg/dm $; \mathrm{d}^{3}=95,00$; $\mathrm{mg} / \mathrm{dm}^{3} ; \mathrm{Al}$ trocável $=0,0 \mathrm{cmol} / \mathrm{dm}^{3}$; $\mathrm{Ca}^{+2}+\mathrm{Mg}=4,75 ; \mathrm{cmol}_{\mathrm{c}} / \mathrm{dm}^{3}$ e matéria orgânica $=11,3 \mathrm{~g} / \mathrm{dm}^{3}$.

Foram avaliadas quinze linhagens de feijão-vagem (Hav 13, Hav 14, Hav 21, Hav 22, Hav 25, Hav 38, Hav 40, Hav 41, Hav 49, Hav 53, Hav 56, Hav 60, Hav 65, Hav 67 e Hav 68) provenientes da AGENCIARURAL, Estação Experimental de Anápolis e oito cultivares (Macarrão Favorito Ag 480, Macarrão Preferido Ag 482, Manteiga Maravilha Ag 481, Teresópolis Ag 484, Macarrão Bragança, Macarrão Trepador Topseed, Macarrão Trepador Hortivale e Macarrão Trepador ISLA).

As plantas foram dispostas em fileiras, tutoradas pelo método de varas cruzadas, no espaçamento de $1,00 \mathrm{~m}$ x 0,20 $\mathrm{m}$, em parcelas com vinte plantas, sendo consideradas úteis apenas dez localizadas nas fileiras centrais. $\mathrm{O}$ delineamento experimental foi o de blocos casualizados com vinte e três tratamentos (quinze linhagens e oito cultivares) e três repetições.

Foram feitas adubações de plantio com 20 t/ha de esterco bovino curtido e seco, $300 \mathrm{~kg} / \mathrm{ha}$ de superfosfato simples e $170 \mathrm{~kg} / \mathrm{ha}$ de cloreto de potássio e, em cobertura, aplicando-se $300 \mathrm{~kg} / \mathrm{ha}$ de sulfato de amônio, 50\% aos 20 dias e $50 \%$ aos 40 dias após a semeadura.

No plantio utilizaram-se três sementes por cova, realizando-se o desbaste quinze dias depois, deixando-se uma planta. Durante a condução da cultura foram realizadas pulverizações à base de Deltametrina 2,5E, para combater a cigarrinha do feijoeiro (Empoasca krameari).

Realizaram-se os tratos culturais normais para a cultura, incluindo irrigação por aspersão, procurando-se fornecer quantidade de água suficiente para o bom desenvolvimento da cultura, além de capinas com auxílio de enxadas, procurando-se manter sempre a cultura livre de plantas invasoras.

Foram obtidos dados de precocidade (número de dias da semeadura à antese das primeiras flores). Nas colheitas, em número de cinco, foram obtidos o número e peso de vagens por parcela que derivaram os dados de produtividade e número de vagens por planta. Como características de qualidade foram avaliados comprimento, diâmetro, peso médio, além da porcentagem de fibras das vagens, conforme a metodologia descrita por Silva (1990). Procedeu-se à análise de variância, comparando-se as médias das características avaliadas pelo teste de Tukey ao nível de 5\% de probabilidade.

\section{RESULTADOS E DISCUSSÃO}

Foram observadas diferenças estatisticamente significativas entre os genótipos para todas as características estudadas (Tabelas 1 e 2).

$\mathrm{O}$ intervalo de tempo entre a semeadura e o início da floração, pode ser empregado como parâmetro para estimar a precocidade em cultivares de vagem. Assim, as linhagens Hav 14, Hav 21, Hav 22, Hav 25, Hav 40, Hav 41, Hav 49, Hav 67 e Hav 68, e todas as cultivares comerciais, foram consideradas tardias por apresentarem maiores intervalos de tempo entre a semeadura e o início da floração.

Os valores obtidos para o comprimento, diâmetro e peso médio de vagens das linhagens Hav 40 e Hav 49 e da cul- tivar Manteiga Maravilha Ag 481 situaram-se dentro do padrão comercial, de acordo com o estabelecido por Tessarioli Neto \& Groppo (1992) e Blanco et al. (1997). A cultivar Teresópolis Ag 484 diferiu significativamente dos demais genótipos; Porém, considerando a característica de vagens grandes e achatadas, poderá sofrer restrições para o mercado local. As linhagens Hav 14, Hav 21, Hav 22, Hav 25, Hav 38, Hav 53, Hav 56, Hav 60, Hav 65, Hav 67, Hav 68 e as cultivares Macarrão Preferido Ag 482, Macarrão Trepador ISLA, Macarrão Favorito Ag 480, Macarrão Bragança, Macarrão Trepador Topseed e Macarrão Trepador Hortivale, apresentaram vagens com características fora dos padrões comerciais (Tabela 1).

As linhagens Hav 21, Hav 22, Hav 25, Hav 38, Hav 41, Hav 49, Hav 68 e as cultivares Macarrão Favorito Ag 480, Macarrão Preferido Ag 482, Manteiga Maravilha Ag 481, Teresópolis Ag 484, Macarrão Trepador Hortivale e Macarrão Trepador Topseed, apresentarem número de vagens por planta dentro do padrão para a espécie. O comportamento das linhagens Hav 21 e Hav 68 com relação ao número de vagens foi semelhante ao observado por Hamasaki et al. (1998) nas condições de Jaboticabal-SP. Em relação a trabalhos realizados em Areia-PB, as linhagens Hav 41, Hav 68 e as cultivares Macarrrão Preferido Ag 482 e Teresópolis Ag 484 apresentaram número de vagens superior aos obtidos por Santos (1999) com a cultivar Macarrão Trepador Topssed e inferior ao obtido por Alves (1999) com a cultivar Macarrão Trepador Hortivale. Já as linhagens Hav 40, Hav 65, Hav 67, Hav 53, Hav 14 e as cultivares Macarrão Bragança e Macarrão Trepador ISLA, apresentaram baixo número de vagens por planta.

As produtividades de vagens obtidas pelas linhagens Hav 25, Hav 41, Hav 13, Hav 21 Hav 49, Hav 68, Hav 22, Hav 38 e pelas cultivares Macarrão Favorito $\mathrm{Ag}$ 480, Macarrão Preferido Ag 482, Manteiga Maravilha Ag 481, Teresópolis Ag 484, Macarrão Trepador Hortivale e Macarrão Trepador Topseed, foram superiores a $25 \mathrm{t} / \mathrm{ha}$, valor da produtividade média nacional (Tessarioli Neto \& Gropp, 1992; Blanco et al., 1997). As linhagens e as cultivares mais produtivas foram as que apresentaram maior número de vagens por planta, fi- 
Tabela 1. Precocidade, comprimento, diâmetro e peso médio de vagens de linhagens e cultivares de feijão-vagem. Areia, UFPB, 1999.

\begin{tabular}{|c|c|c|c|c|}
\hline Linhagens e cultivares & Precocidade $^{1}$ & $\begin{array}{c}\text { Comprimento } \\
\text { (cm) }\end{array}$ & $\begin{array}{l}\text { Diâmetro } \\
\text { (cm) }\end{array}$ & $\begin{array}{l}\text { Peso médio } \\
\text { (g) }\end{array}$ \\
\hline Hav 13 & $38 \quad d$ & $17,0 \quad b c$ & $1,20 \quad b c$ & $9,8 \mathrm{abc}$ \\
\hline Hav 14 & 48 a & 15,7 & $1,16 \mathrm{bc}$ & $10,0 \mathrm{abc}$ \\
\hline Hav 21 & $43 \mathrm{abcd}$ & 15,6 & $1,30 \mathrm{abc}$ & $9,0 \quad b c$ \\
\hline Hav 22 & 49 a & 16,5 & $1,13 \mathrm{bc}$ & 7,6 bc \\
\hline Hav 25 & $47 a b$ & 15,1 & 1,03 & $7,3 \mathrm{bc}$ \\
\hline Hav 38 & $40 \mathrm{bcd}$ & 16,3 & 1,16 & $9,9 \mathrm{abc}$ \\
\hline Hav 40 & $43 \mathrm{abcd}$ & $20,1 a b$ & 1,16 bc & $9,6 a b c$ \\
\hline Hav 41 & 49 a & 17,4 bc & 1,10 & 7,9 bc \\
\hline Hav 49 & $46 \mathrm{abc}$ & $21,9 a$ & 1,20 & $11,4 a b$ \\
\hline Hav 53 & $38 \quad d$ & $16,1 \quad c$ & 1,10 & 9,1 bc \\
\hline Hav 56 & $40 \mathrm{bcd}$ & 14,4 & 1,16 & $8,0 \mathrm{bc}$ \\
\hline Hav 60 & $39 \quad d$ & 15,1 & 1,13 & $8,2 \mathrm{bc}$ \\
\hline Hav 65 & $40 \mathrm{~cd}$ & 15,5 & 1,33 & 9,0 bc \\
\hline Hav 67 & $43 \mathrm{abcd}$ & 14,8 & 1,16 & $6,9 \quad c$ \\
\hline Hav 68 & $45 \mathrm{abc}$ & 15,5 & 1,13 & 9,1 bc \\
\hline Macarrão Favorito Ag 480 & $48 a$ & 15,1 & 1,16 bc & 8,0 bc \\
\hline Macarrão Preferido Ag 482 & $48 a$ & $16,3 \quad c$ & $1,13 \mathrm{bc}$ & 8,2 bc \\
\hline Manteiga Maravilha Ag 481 & 48 a & $21,2 \mathrm{a}$ & $1,36 a b$ & $10,8 a b c$ \\
\hline Teresópolis Ag 484 & 48 a & $23,5 \mathrm{a}$ & $1,50 \mathrm{a}$ & $13,7 \mathrm{a}$ \\
\hline Macarrão Bragança & $48 a$ & $15,1 \quad c$ & $1,06 \mathrm{bc}$ & $9,6 \mathrm{abc}$ \\
\hline Macarrão Trepador ISLA & $48 a$ & 15,4 & $1,16 \mathrm{bc}$ & $9,7 \mathrm{abc}$ \\
\hline Macarrão Trepador Hortivale & $47 a b$ & 14,1 & $1,13 \mathrm{bc}$ & $9,5 \mathrm{bc}$ \\
\hline Macarrão Trepador Topseed & $48 \mathrm{a}$ & 14,9 & $1,03 \quad \mathrm{c}$ & 8,7 bc \\
\hline CV $(\%)$ & 3,9 & 7,7 & 7,2 & 14,4 \\
\hline
\end{tabular}

Médias seguidas da mesma letra, na coluna, não diferem entre si pelo teste de Tukey ao nível de 5\% de probabilidade.

${ }^{1}$ Número de dias da semeadura à antese das primeiras flores.

cando evidenciado uma provável correlação positiva entre esta característica e a produtividade. As linhagens Hav 40, Hav 65, Hav 53, Hav 67, Hav 56, Hav 14, Hav 60 e as cultivares, Macarrão Bragança e Macarrão Trepador ISLA, apresentaram produtividade abaixo da média nacional (Tabela 2).

$O$ teor de fibra nas vagens das linhagens e cultivares variou de 0,71 a $1,60 \%$ (Tabela 2). A linhagem Hav 49 foi a mais fibrosa, porém, estatisticamente, diferente apenas de Hav 41 e Hav 60 . O resultado para a Hav 49 foi o oposto do obtido por Hamasaki et al. (1998), em Jaboticabal, onde essa linhagem foi a menos fibrosa entre os genótipos avaliados. Isto pode ter sido em função de diferentes estádios de desenvolvimento das vagens colhidas nos dois locais, já que Silbernagel \& Drake (1978) de- monstraram que o tamanho das sementes pode ser utilizado na elaboração de um índice de qualidade relativo ao teor de fibras. A linhagem Hav 41, foi a menos fibrosa, estando este resultado de acordo com o observado por Hamasaki et al. (1998) em Jaboticabal. No entanto, o teor de fibra na linhagem Hav 41 foi estatisticamente igual às linhagens Hav 38, Hav 13, Hav 21, Hav 14, Hav 25, Hav 68, Hav 56, Hav 53, Hav 60, e às cultivares Macarrão Trepador Topseed, Macarrão Favorito Ag 480, Macarrão Trepador ISLA, Macarrão Preferido Ag 482 e Macarrão Trepador Hortivale. A linhagem Hav 49 apresentou boa produtividade e boa aparência das vagens (Tabela 2).

Como para o produtor de hortaliças é desejável a utilização de cultivares adaptadas às suas condições edafoclimáticas
(Peixoto et al., 1993), as cultivares Macarrão Preferido Ag 482 e Manteiga Maravilha Ag-481 por serem as mais produtivas entre as de melhor qualidade, podem ser indicadas como alternativas ao produtor, inclusive com maior possibilidade de lucro, considerando-se as cultivares Macarrão Trepador Topseed e Macarrão Trepador Hortivale como padrão comercial de vagens no mercado de Areia-PB. As cultivares Macarrão Favorito Ag 480 e Macarrão Bragança, além das linhagens Hav 22, Hav 38, Hav 41 e Hav 68 também poderão atender a esse propósito, visto que apresentaram produtividade e características de vagens satisfatórias. A cultivar Teresópolis Ag 484, a mais produtiva, tem sua indicação para cultivo condicionada a estudos de mercado, pelo fato de apresentar vagens fora do padrão comercial nessa região. 
Tabela 2. Número médio de vagens por planta, produtividade de vagens no ponto comercial e porcentagem de fibras nas vagens em linhagens e cultivares de feijão-vagem. Areia, UFPB, 1999.

\begin{tabular}{|c|c|c|c|}
\hline Linhagens e cultivares & Vagens por planta $\left(n^{0}\right)$ & $\begin{array}{c}\text { Produtividade } \\
\text { (t/ha) }\end{array}$ & Fibras (\%) \\
\hline Hav 13 & 31,4 bcd efg & 25,6 bcd & 1,27 abcd \\
\hline Hav 14 & $23,6 \quad \mathrm{fg}$ & $20,7 \mathrm{bcd}$ & 1,19 abcd \\
\hline Hav 21 & 35,5 abcdefg & $27,6 \mathrm{bcd}$ & $1,25 \mathrm{abcd}$ \\
\hline Hav 22 & $49,1 \mathrm{abc}$ & $31,6 \mathrm{bcd}$ & $1,37 a b c$ \\
\hline Hav 25 & 40,5 abcdefg & $26,0 \mathrm{bcd}$ & $1,15 \mathrm{abcd}$ \\
\hline Hav 38 & 43,8 abcdef & $35,0 \mathrm{bc}$ & $1,28 \mathrm{abcd}$ \\
\hline Hav 40 & 29,5 cdefg & $24,1 \mathrm{bcd}$ & $1,53 a b$ \\
\hline Hav 41 & $48,1 \mathrm{abcd}$ & $31,5 \mathrm{bcd}$ & $0,71 \quad d$ \\
\hline Hav 49 & 37,0 abcdefg & $35,7 \mathrm{bc}$ & $1,60 \mathrm{a}$ \\
\hline Hav 53 & 26,5 efg & $20,8 \mathrm{bcd}$ & $0,91 \mathrm{bcd}$ \\
\hline Hav 56 & 33,3 abcdefg & $22,6 \mathrm{bcd}$ & $1,05 \mathrm{abcd}$ \\
\hline Hav 60 & 33,4 abcdefg & $24,0 \mathrm{bcd}$ & $0,84 \mathrm{~cd}$ \\
\hline Hav 65 & 28,7 defg & $20,8 \mathrm{bcd}$ & $1,45 a b c$ \\
\hline Hav 67 & $27,6 \quad$ efg & $15,9 \quad d$ & $1,40 a b c$ \\
\hline Hav 68 & 39,9 abcdefg & $30,5 \mathrm{bcd}$ & 1,09 abcd \\
\hline Macarrão Favorito Ag 480 & 45,8 abcde & $30,3 \mathrm{bcd}$ & $1,20 \mathrm{abcd}$ \\
\hline Macarrão Preferido Ag 482 & $50,4 a b$ & $39,1 \quad b$ & $1,05 \mathrm{abcd}$ \\
\hline Manteiga Maravilha Ag 481 & 38,8 abcdefg & $36,2 \quad b$ & $1,53 a b$ \\
\hline Teresópolis Ag 484 & $53,4 \mathrm{a}$ & $58,5 \mathrm{a}$ & $1,38 a b c$ \\
\hline Macarrão Bragança & 24,1 & $20,2 \mathrm{bcd}$ & $1,38 \mathrm{abc}$ \\
\hline Macarrão Trepador ISLA & 21,1 & $17,2 \mathrm{~cd}$ & 1,15 abcd \\
\hline Macarrão Trepador Hortivale & 34,9 abcdefg & $26,5 \mathrm{bcd}$ & $1,00 \mathrm{abcd}$ \\
\hline Macarrão Trepador Topseed & 40,1 abcdefg & $28,8 \mathrm{bcd}$ & $1,23 \mathrm{abcd}$ \\
\hline $\mathrm{CV}(\%)$ & 17,8 & 21,3 & 16,0 \\
\hline
\end{tabular}

Médias seguidas da mesma letra, na coluna, não diferem entre si pelo teste de Tukey ao nível de $5 \%$ de probabilidade

\section{AGRADECIMENTOS}

Os autores agradecem aos agentes em Agropecuária, Francisco de Castro Azevedo, José Barbosa de Souza, Francisco Soares de Brito, Francisco Silva do Nascimento e Expedito de Souza Lima que viabilizaram a execução dos trabalhos de campo.

\section{LITERATURA CITADA}

ALVES, E.U. Produção e qualidade de sementes de feijão-vagem em função de fontes e doses de matéria orgânica. Areia: CCA-UFPB, 1999. 108 p. (Tese mestrado).

BLANCO, M.C.S.G.; GROPPO, G.A.; TESSARIOLI NETO, J. Feijão-vagem (Phaseolus vulgaris L.) Manual Técnico das Culturas, Campinas, n. 8, p. 63-65, 2a ed., 1997.
FILGUEIRA, F.A.R. Manual de Olericultura: cultura e comercialização. 2 ed. São Paulo, Agronômica Ceres, 338 p. 1982.

HAMASAKI, R.I.; BRAZ, L.T.; PURQUERIO. L..F.V.; PEIXOTO, N. Comportamento de novas cultivares de feijão-vagem em Jaboticabal-SP. CONGRESSO BRASILEIRO DE OLERICULTURA, 38., 1998, Petrolina. Resumo... Petrolina: SOB, 1998.

NADAL, R.; GUIMARÃES, D.R.; BIASI, J.; PINHEIRO, S.L.J.; CARDOSO, V.T.M., Olericultura em Santa Catarina: aspectos técnicos e econômicos. Florianópolis: EMPASC, 1986. p. $130-136$

PEIXOTO, N.; SILVA, L.O.; THUNG, M.D.T.; SANTOS, G. Produção de sementes de linhagens e cultivares arbustivas de feijão-vagem em Anápolis. Horticultura Brasileira, Brasilia, v. 11, n. 2, p. 151-152, 1993.

PEIXOTO, N.; THUNG, M.D.T.; SILVA, L.O.; FARIAS, J.G.; OLIVEIRA, E.B.; BARBEDO, A.S.C.; SANTOS, G. Avaliação de cultivares arbustivas de feijão-vagem, em diferentes ambientes do Estado de Goiás. Goiânia-GO. EMATER-GO Assessoria de Comunicação Social, 1997 (Boletim de Pesquisa 01).
SANTOS, G.M. Produção e qualidade do feijãovagem em função de fontes e doses de matéria orgânica. Areia: CCA-UFPB, 1999. 98 p. (Tese mestrado)

SILBERNAGEL, M.J.; DRAKE, S.R. Seed index, an estimate of snap bean quality. American Society for Horticultural Science. v. 103, n. 2, p. 257-260, 1978.

SILVA, D.J.; Análise de Alimentos (Métodos químicos e biológicos), $2^{\mathrm{a}}$ ed. 165 p. 1990.

TESSARIOLI NETO, J.; GROPPO, G.A.. A cultura do fejão-vagem, Boletim técnico CATI., Campinas-SP, n. 212, p. 1-12, 1992.

VIGGIANO, J. Produção de Sementes de feijãovagem. In: CASTELLANE, P.D. NICOLOSI, W. M, HASEGAWA, M., coord. Produção de sementes de hortaliças. Jaboticabal-SP: Faculdade de Ciências Agrárias e Veterinárias/ Fundação de Estudos e Pesquisas em Agronomia, Medicina Veterinária e Zootecnia, 1990, p.127-140. 